\title{
New Configurations of Low-Cost Dual-Polarized Printed Antennas for UWB Arrays
}

\author{
Guido Valerio, ${ }^{1}$ Simona Mazzocchi, ${ }^{2}$ Alessandro Galli, ${ }^{2}$ \\ Matteo Ciattaglia, ${ }^{3}$ and Marco Zucca $^{3}$ \\ ${ }^{1}$ Institut d'Electronique et des Télécommunications de Rennes (IETR), UMR CNRS 6164, Université de Rennes 1,
35042 Rennes Cedex, France
${ }^{2}$ Department of Information Engineering, Electronics and Telecommunications, Sapienza University of Rome, 00184 Rome, Italy
${ }^{3}$ SELEX S.I. S.p.A., Sistemi Radianti, 00131 Rome, Italy
}

Correspondence should be addressed to Guido Valerio, guido.valerio@univ-rennes1.fr

Received 15 August 2011; Revised 26 November 2011; Accepted 6 December 2011

Academic Editor: Athanasios Panagopoulos

Copyright () 2012 Guido Valerio et al. This is an open access article distributed under the Creative Commons Attribution License, which permits unrestricted use, distribution, and reproduction in any medium, provided the original work is properly cited.

\begin{abstract}
A novel class of structures is proposed to realize ultra-wide-band radiating elements for large arrays, providing dual polarization, beam scanning, and compact and inexpensive realization based on suitable rhombic arrangements of dipoles printed on low-cost layered substrates. In a first implementation, four rhombic shapes, orthogonally placed on the same layer, provide two orthogonal polarizations. In a second implementation, the two polarizations are excited by two rhombic shapes printed on two different layers in a stacked-patch-like arrangement. This latter structure leads to a better lateral shielding of the single radiating element, in order to reduce mutual interactions among adjacent elements in array environment. The behavioral features of these antennas have been tested with various parametric analyses. Practical aspects have been addressed such as the choice of appropriate feeding and of commercially available dielectric layers. The resulting antennas are matched at the input ports in an extremely wide range of frequencies $(5-25 \mathrm{GHz})$, covering various microwave applications, such as aircraft surveillance, weather polarimetric radars, and control and communications systems. Good radiating features, in terms of pattern shape and gain, are observed in a large band of frequencies. The basic scanning performance of large and small array configurations is finally investigated.
\end{abstract}

\section{Introduction}

The development of ultra-wide-band (UWB) antennas is recently providing new solutions for the design of innovative, versatile, and economical radar and communication systems. This class of structures is in fact gaining increasing attractiveness for the enhanced features of the wideband signals, for instance in applications to imaging, surveillance, and highcapacity communications [1-4].

In impulse radar techniques, UWB antennas allow to transmit and receive short-pulsed signal waveforms without significant distortions. In the field of wireless communications, the demand for wideband radiating elements grows up as well. Furthermore, the dramatic reduction of costs related to a single equipment for multiple applications (communications, surveillance, weather, etc.) is possible if a suitably wide band of frequency can be used and efficiently treated by the same radiating element [5-9].
Many UWB antennas based on printed configurations have been proposed in the past years $[3,10]$. They are currently of great interest due to various advantages $[11,12]$ : low cost, light weight, compactness and conformability, compatibility with printed-circuit-board (PCB) technologies, and high reproducibility in the realization of large array radars. The most common implementation is based on monopole structures, having bidirectional radiating properties. While a reflecting ground plane could be introduced to maximize the gain in the required half plane, this usually introduces resonant behaviors in the currents on the metalization, thus significantly reducing the useful operational bandwidth of the structure.

More complex configurations should be designed if grounded structures are of interest: for example, the use of parasitic elements can induce resonances at different frequencies thus enlarging the total available band 
[13-15]. In this paper, novel configurations of printed dipoles arranged in rhombic shapes are proposed to radiate over a very wide band of frequency with applications to array radars and UWB communication systems, following a preliminary design presented in [16]. The frequency ranges of interest here are mainly for the $\mathrm{C}, \mathrm{X}, \mathrm{K}_{u}$, and $\mathrm{K}$ bands (approximately between 5 and $25 \mathrm{GHz}$ ). Suitable scaling of such structures anyway allows for covering other possible microwave ranges for UWB applications. The basic principles for the new types of radiators are introduced in Section 2. Specific solutions are considered here for dualpolarization applications. In Section 3, a first topology is described, composed by four coplanar rhombic elements (see Figure 1). In Section 4, a laterally shielded structure, feasible for implementation in an array environment, is presented: two rhombic elements are printed on different layers, in a simple stacked configuration, each providing a linear polarization orthogonal to each other (see Figure 2). Extensive results are shown for both the structures, to show the UWB matching features through the relevant input parameters, and the radiating characteristics of the single radiating elements and of various array configurations.

\section{Basic Structures}

The two structures presented here are based on various arrangements of printed dipoles of different lengths forming approximately rhombic-shaped elements (see Figures 1 and 2 ). The central patch is the longest and can be fed at its ends with one or two probes; the side patches, being six or eight depending on the structure, have smaller dimensions and are parasitic. To further increase the bandwidth, the dipoles are printed on a three-layer structure, designed with low-cost commercially available dielectric substrates. In the present analysis, the lower and the upper layers are duroid RT 5880 Rogers substrates with relative permittivity $\varepsilon_{\mathrm{r}, 1}=2.2$ and dielectric loss tangent $\tan \delta_{1}=9.0 \cdot 10^{-4}$, the middle layer is an FR4 epoxy substrate with $\varepsilon_{\mathrm{r}, 2}=4.4$ and dielectric loss tangent $\tan \delta_{2}=200.0 \cdot 10^{-4}$. It should be pointed out that, even if loss effects can become nonnegligible (particularly in higher-frequency ranges); in this case the choice of such inexpensive commercial substrates is mainly related to the strong reduction of costs in the implementation of large arrays of elements, exploiting also well-established manufacturing printed-circuit techniques of PCB [17]. A number of other materials having similar electric parameters but reduced losses can be chosen if higher efficiency is desired (e.g., for the internal layer a dielectric such as TMM4, having a loss tangent one order of magnitude lower than FR4 can be employed). Some compared results concerning the influence of such losses on the gain and efficiency of these antennas will be presented next. In the following analyses, also nonideal effects of the metalization are taken into account, considering finite-conductivity strips made of copper $\left(\sigma=5.8 \cdot 10^{7} \mathrm{~S} / \mathrm{m}\right.$, $\left.\mu_{r}=0.999991\right)$ having nonzero thickness $\left(10^{-6} \mathrm{~m}\right)$. A single rhombic shape mainly provides a field linearly polarized, with components related to the direction of the relevant dipoles. The dual polarizations can thus be obtained using

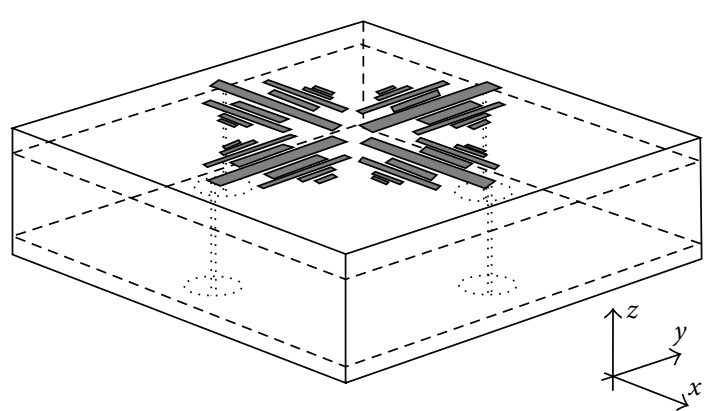

(a)

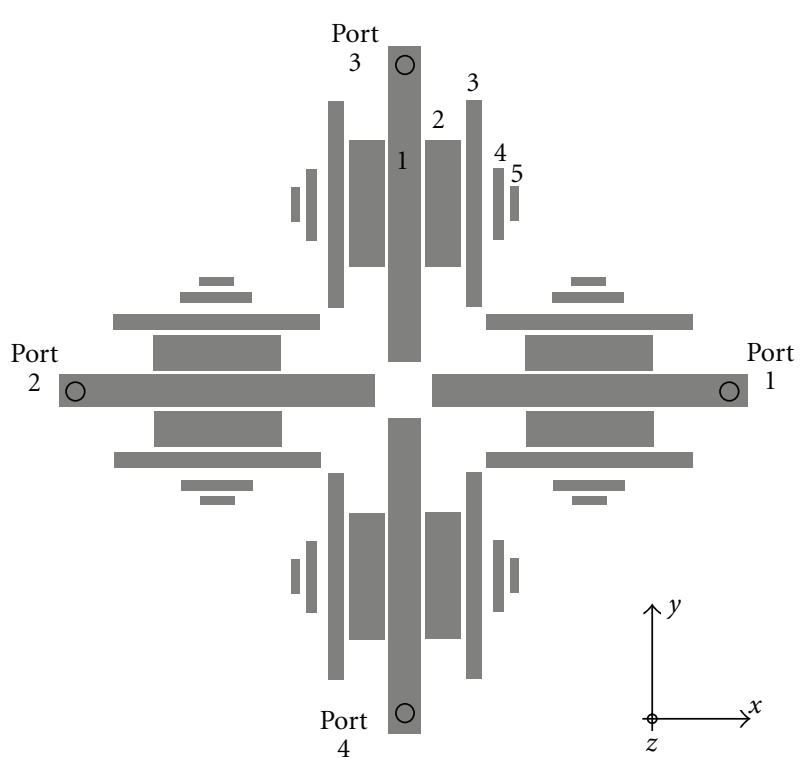

(b)

Figure 1: Geometry of the radiating element under analysis in Section 3, based on four rhombic coplanar elements printed on the top of a three-layer configuration. (a) 3D view of the antenna. (b) Upper view of the structure. Lowest substrate: thickness $h=$ $1.5 \mathrm{~mm}$, relative dielectric constant $\varepsilon_{\mathrm{r}}=2.2$, loss tangent $\tan \delta=$ $9 \cdot 10^{-4}$ (duroid RT 5880 Rogers). Middle substrate thickness $h=$ $7.5 \mathrm{~mm}$, relative dielectric constant $\varepsilon_{\mathrm{r}}=4.4$, loss tangent $\tan \delta=$ $200 \times 10^{-4}$ (FR4 epoxy). Uppermost substrate thickness $h=2 \mathrm{~mm}$, relative dielectric constant $\varepsilon_{\mathrm{r}}=2.2$, loss tangent $\tan \delta=9 \cdot 10^{-4}(\mathrm{RT}$ 5880 Roger). Overall unit-cell dimension $43 \times 43 \mathrm{~mm}$. Geometrical parameters of the patches: $w_{1}=1.9 \mathrm{~mm}, l_{1}=17.3 \mathrm{~mm}, w_{2}=2 \mathrm{~mm}$, $l_{2}=7 \mathrm{~mm}, w_{3}=1 \mathrm{~mm}, l_{3}=11.3 \mathrm{~mm}, w_{4}=0.6 \mathrm{~mm}, l_{4}=4 \mathrm{~mm}$, $w_{5}=0.4 \mathrm{~mm}$, and $l_{5}=2 \mathrm{~mm}\left(w_{i}\right.$ and $l_{i}$ are the width and the length of the $i$ th patch, resp.). Diameter of the probe $d=0.86 \mathrm{~mm}$.

a couple of rhombic elements, mutually rotated of $90^{\circ}$. The relevant features of two possible rhombic arrangements are analyzed in detail in the next two sections.

\section{Unshielded Antenna with Coplanar Dual Rhombic Elements}

3.1. Structure. A fully dual-polarized radiating element can be obtained by arranging four rhombic shapes, printed on the top of the three-layer structure described in the previous 


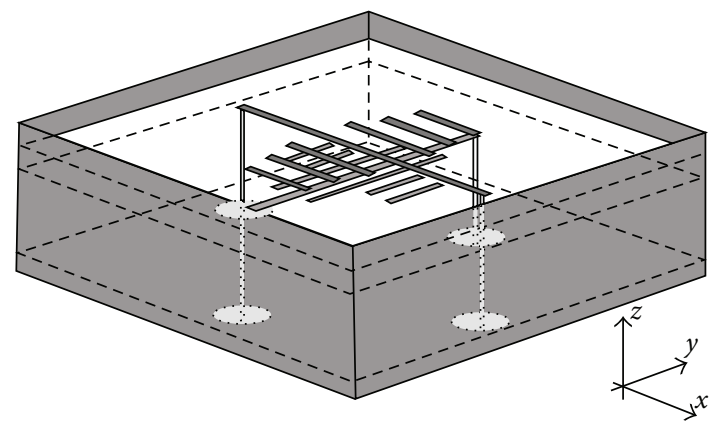

(a)

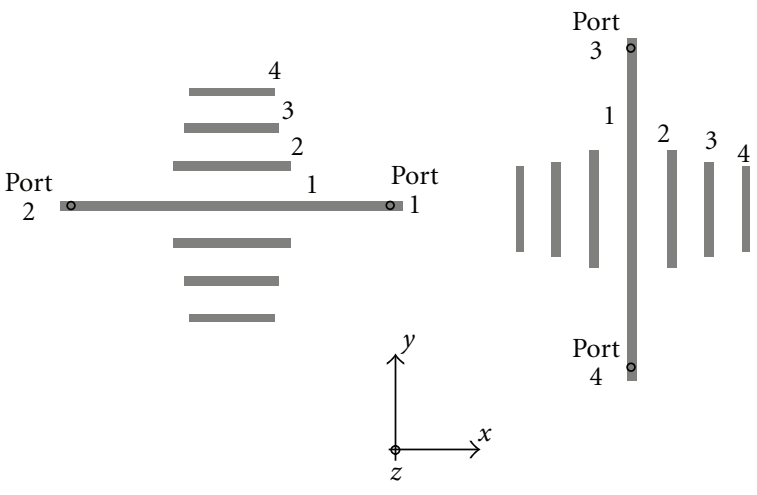

(b)

FIGURE 2: Geometry of the radiating element under analysis in Section 4, based on another rhombic element printed on the top layer, and a rhombic element printed on the second layer. (a) 3D view of the antenna. (b) Upper view of the structure. Same threelayer configuration as in Figure 1. Geometrical parameters of the patches of the upper element: $w_{1}=1 \mathrm{~mm}, l_{1}=29.6 \mathrm{~mm}, w_{2}=$ $0.8 \mathrm{~mm}, l_{2}=10 \mathrm{~mm}, w_{3}=0.6 \mathrm{~mm}, l_{3}=8 \mathrm{~mm}, w_{4}=0.6 \mathrm{~mm}$, and $l_{4}=7 \mathrm{~mm}$. The diameter of the probes feeding the upper element is $d=0.24 \mathrm{~mm}$. Geometrical parameters of the patches of the lower element: $w_{1}=0.6 \mathrm{~mm}, l_{1}=26.8 \mathrm{~mm}, w_{2}=0.6 \mathrm{~mm}, l_{2}=15.6 \mathrm{~mm}$, $w_{3}=0.6 \mathrm{~mm}, l_{3}=6 \mathrm{~mm}, w_{4}=0.6 \mathrm{~mm}$, and $l_{4}=6 \mathrm{~mm}$. ( $w_{i}$ and $l_{i}$ are the width and the length of the $i$ th patch, resp.) The diameter of the probes feeding the lower element is $d=0.4 \mathrm{~mm}$. The size of the box is $40 \mathrm{~mm}$.

section, as in Figure 1. The central patch of each rhombic element is fed with a probe at its outer end, and opposite patches are fed with signals having the same magnitude and opposite sign. With this geometrical arrangement, one couple of opposite rhombic elements provides one linear polarization, and the other couple of opposite elements, rotated of $90^{\circ}$, provides the orthogonal polarization. If the same signal is radiated through both the polarizations, a general elliptical polarization can be obtained, by suitably tuning the relevant phase shifts among the input probes. In particular, a circular polarization can be obtained by imposing a $90^{\circ}$ phase shift between adjacent elements.

The antenna is designed to work in a very wide frequency range, possibly greater than $\mathrm{C}-\mathrm{X}$ bands $(4-12 \mathrm{GHz})$ in the case of interest. Its transverse dimensions of the element are approximately $4 \mathrm{~cm}$, leading to a possible compact implementation in array at these frequencies. Different operational requirements are given at lower and at higher frequencies. Specifically, at lower frequencies both a good matching and a regular radiation pattern are required, whilst at higher frequencies, because of a different utilization of the control system, a good matching only can be sufficient. The dimensions of the various patches have been designed in order to reach an optimum input matching in the working wide band $5-25 \mathrm{GHz}$, as shown in next subsection. Further simulations, here omitted for brevity, would show that the input matching is preserved also at frequencies higher than $30 \mathrm{GHz}$, even though the radiation patterns are less regular as the frequency increases.

Beyond these advantages in terms of extremely wide band, low-profile and low-cost realization, and radiation pattern regularity, a key promising feature of this structure is also the dynamic conformability of its radiation pattern, due to the use of a pair of probes for the feeding of each polarization. For instance, if an array of elements is placed on a conformable surface, a fine tuning of the pointing angle can be easily obtained by an appropriate tailoring of the feeding network.

3.2. Analysis and Results. A throughout optimization procedure, involving the dimensions of the patches and the positions of the feeding probes, has been carried out with the software package "ModeFrontier" [18], with the aim of achieving the best input matching in the desired frequency band. The optimization has been performed at first on a single rhombic shape and then refined with the full fourrhombus structure. The algorithm used is a multiobjective genetic algorithm with multisearch elitism for enhanced robustness [19]. Its objectives were chosen as the conditions $\left|S_{i j}\right|<-10 \mathrm{~dB}$ at $5 \mathrm{GHz}$ for any $i, j$, and the variables were all the dimensions and positions of the various patches.

The results shown here have been computed with the time-domain solver of the high-frequency electromagnetic CAD "CST Studio 2010" [20]. The layered substrate is assumed laterally unbounded, and a single radiating element, composed by four rhombic shapes, is considered as in Figure 1. The input ports are modeled as coaxial cables, fed with proper phase shifts. The feeding network providing these shifts among the different ports is not simulated here and will be object of future work. The CST model is discretized with hexahedral cells of average dimension $\lambda / 10$; open conditions are placed at the side boundary of the cell, thus assuming a laterally unbounded substrate; extra space is added in the top half plane in order to accurately estimate the radiation patterns. Waveguide ports are defined at the coaxial cables and excited with the fundamental TEM mode.

Based on the data sheets of the electromagnetic parameters of these materials as a function of the frequency (if available), the dispersive effects can be taken properly into account in the simulations. The relevant analysis shows anyway that these dispersive effects do not change sensitively the performance of our antennas in the ranges of interest.

Due to the geometrical symmetries, the input features of the antenna are described by three different scattering parameters. In Figure 3, the magnitude in $\mathrm{dB}$ of these three 


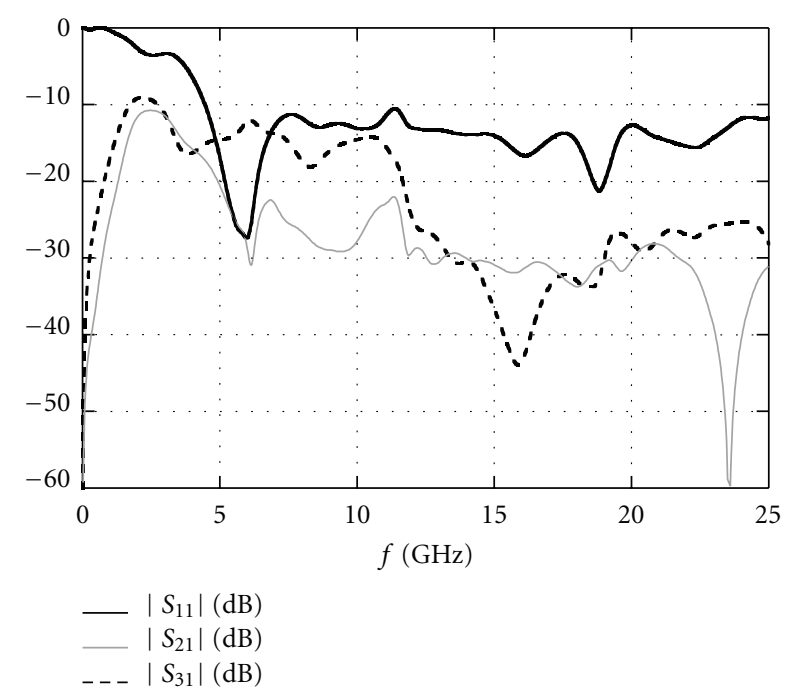

Figure 3: Antenna of Figure 1. Magnitude of the scattering coefficients of the radiating element fed by the four probes. Reflection coefficient $\left|S_{11}\right|$ at the input port 1 (black line) and transmission coefficients $\left|S_{21}\right|$ from port 1 to port 2 (gray line) and from port 1 to port 3 (dashed black line).

parameters is shown in the frequency band considered, from dc to $25 \mathrm{GHz}$. The reflection coefficient at one input cable is shown in black solid line (due to the symmetry of the structure, the cable considered is arbitrary, since $S_{11}=S_{22}=$ $S_{33}=S_{44}$ ). The coupling between opposite ports (e.g., $\left.\left|S_{21}\right|\right)$ is shown in gray solid line, and the coupling between adjacent ports (e.g., $\left|S_{31}\right|$ ) is shown in black dashed line. The operational frequency band where the parameters are under the conventional threshold of $-10 \mathrm{~dB}$ is extremely wide: in the figure, the band $5-25 \mathrm{GHz}$ is well matched, but further simulations would show a good matching also beyond $30 \mathrm{GHz}$, even though, as said, the radiation patterns tend to deteriorate as the frequency increases. In Figure 4(a), the gain is shown on the principal plane $\varphi=0^{\circ}$ at different frequencies from $5 \mathrm{GHz}$ to $25 \mathrm{GHz}$ if the ports 1 and 2 are fed with common amplitude and opposite phase: by symmetry, the electric field has only a $\theta$-component. In Figure $4(\mathrm{~b})$, the gain is shown on the same plane if the ports 3 and 4 are fed with common amplitude and opposite phase: in this case, by symmetry, the electric field has only a $\varphi$-component. Good radiation features are found in the lower band of frequency, while the pattern tends to deform at higher frequencies as expected.

The features of the maximum gain are resumed in Table 1, considering their values with respect to the isotropic radiator $(\mathrm{dBi}$ and the relevant beam angular locations on the different planes). In the same table, maximum gain and radiation efficiencies are also presented with reference to the alternative choice of the TMM4 for the middle layer (with reduced losses, see Section 2 for details). As shown, acceptable results are achieved even with the lossy substrate, while significant improvements in the efficiency can be obtained at higher frequencies with the use of a lower-loss substrate.

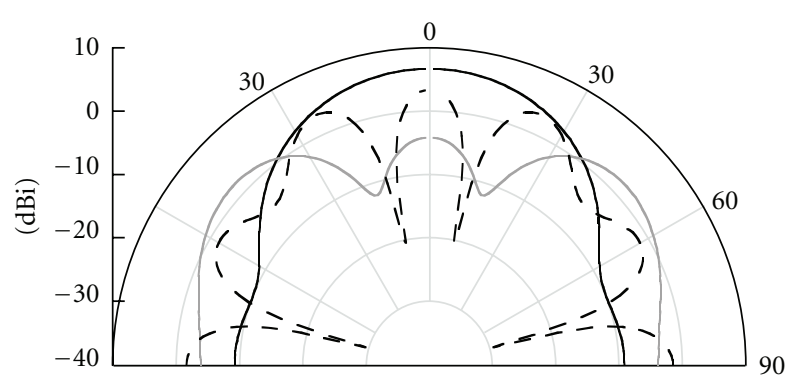

(a)

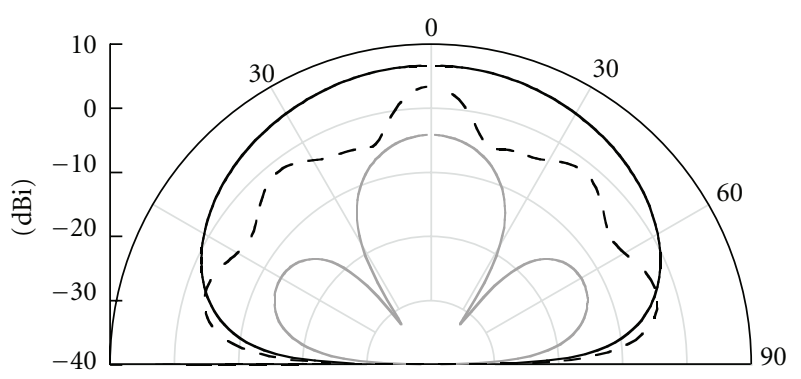

(b)

FIgURE 4: Antenna in Figure 1. Gain in polar form on the principal elevation plane $\phi=0^{\circ}$ of the single element at different frequencies in the operational bandwidth 5-25 GHz: $5 \mathrm{GHz}$ (solid black line), $10 \mathrm{GHz}$ (solid gray line), $20 \mathrm{GHz}$ (dashed black line). (a) Ports 1 and 2 are fed, exciting a field along the $\theta$ direction. (b) Ports 3 and 4 are fed, exciting a field along the $\varphi$ direction.

\section{Shielded Antenna with Stacked Dual Rhombic Elements}

4.1. Structure. Once the antenna described in the previous section is introduced in an array environment, the UWB input performance of the array could deteriorate due to strong mutual coupling among adjacent elements. To overcome such a problem, a lateral shielding of the single radiating element can be implemented with metallic walls. Such a configuration also enables us to reduce adverse effects related to possible launching of surface and leaky waves in the layered structure $[11,12]$. As an example of this approach, an alternative shielded radiating element is presented here, based on the same rhombic printed shapes described above, but with elements placed on different layer interfaces.

In this case, shown in Figure 2, each couple of opposite rhombic elements is here replaced by a single rhombus, whose central larger patch is fed by two different probes at its ends, carrying signals with common amplitude and opposite phase. With this original feeding structure involving two probe elements on the opposite sides of each main strip, it is noted that, by properly choosing the phase shift between the feeders, it is also possible to achieve a straightforward control of the current configuration excited on the strips, thus suitably influencing the directional features of the radiated beam. In this structure, the two orthogonal polarizations are then provided by two different rhombic elements, rotated by $90^{\circ}$ and printed on two different layers: the dipoles of 
TABLe 1: Maximum gain: on $\varphi=0^{\circ}$.

\begin{tabular}{cccccc}
\hline$f(\mathrm{GHz})$ & Direction $(9)$ & Max gain & Efficiency & Max gain (low loss) & Efficiency (low loss) \\
\hline 5 & $\vartheta=0^{\circ}$ & $6.6 \mathrm{dBi}$ & $92.9 \%$ & $7.1 \mathrm{dBi}$ & $99 \%$ \\
10 & $\vartheta=90^{\circ}, \varphi=54^{\circ}$ & $3.8 \mathrm{dBi}$ & $78.7 \%$ & $4.5 \mathrm{dBi}$ & $96.6 \%$ \\
15 & $\vartheta=45^{\circ}, \varphi=22^{\circ}$ & $3.2 \mathrm{dBi}$ & $80.0 \%$ & $3.7 \mathrm{dBi}$ & $96.8 \%$ \\
20 & $\vartheta=38^{\circ}, \varphi=24^{\circ}$ & $3.7 \mathrm{dBi}$ & $64.1 \%$ & $4.9 \mathrm{dBi}$ & $92.7 \%$ \\
25 & $\vartheta=90^{\circ}, \varphi=9^{\circ}$ & $5.5 \mathrm{dBi}$ & $68.4 \%$ & $6.2 \mathrm{dBi}$ & $93.3 \%$ \\
\hline
\end{tabular}

one rhombus are printed on the uppermost layer and the dipoles of the other rhombus on the middle layer. Also in this case the box has transverse dimensions of $4 \mathrm{~cm}$, leading to good performance in a possible array implementation. On the other hand, the distance between the structure (i.e., strips and cables) and the lateral boundaries is greater in this new configuration than in the previous one, leading to an easier input matching also in the shielded configuration.

Again, the dimensions of the various patches have been optimized in order to yield the optimum input matching in the frequency band 5-25 GHz. Of course, considering such a different arrangement of the rhombic shapes, rather different geometrical parameters have been reached in this second structure as optimum values.

4.2. Analysis and Results. The results shown here have been obtained through full-wave simulations of the final optimized stacked and shielded structure again with the time-domain solver of CST Studio.

In Figure 5, the magnitude in $\mathrm{dB}$ of the three scattering parameters of the shielded configuration is shown; also with this structure, a good input matching is reached in the very wide frequency band $5-25 \mathrm{GHz}$. The reflection coefficient at port 1 (i.e., feeding the upper rhombic shape) is shown in solid black line, the reflection coefficient at port 3 (i.e., feeding the lower rhombic shape) is shown in solid gray line, while the coupling between the rhombic shapes is shown in dashed black line.

The radiation patterns on the principal planes are shown again for various values of the frequency. In Figure 6(a), the gain is shown on the principal plane $\varphi=0^{\circ}$, when probes 1 and 2 feed the upper element with signals with common magnitude and $180^{\circ}$ phase shift. The electric field is in this case polarized along the $\theta$ direction by symmetry. In Figure 6(b), the gain is shown on the same plane when the lower element is fed through probes 3 and 4 , with common magnitude and $180^{\circ}$ phase shift. A dual polarization is radiated with respect to the previous result, the electric field being polarized along the $\varphi$ direction. As seen, fairly regular radiation patterns are observed for both polarizations, in particular in the lower part of frequency. Since this configuration is studied with an "open add space" lateral boundary in CST, an estimation of backlobes is also present in the results.

The gain on the other principal plane $\varphi=90^{\circ}$ is shown in Figure 7. The upper rhombic shape is fed in Figure 7(a), while the lower rhombic shape is fed in Figure 7(b).

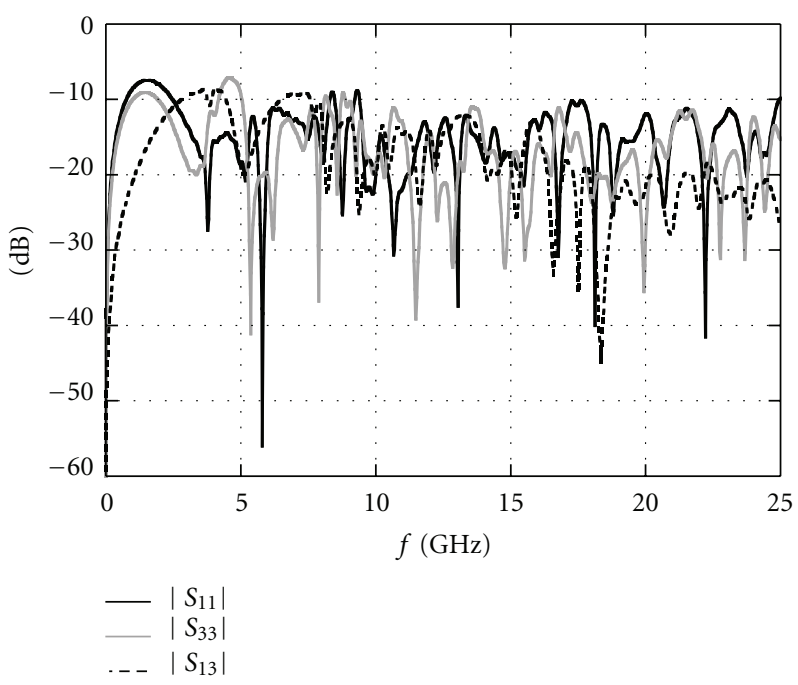

Figure 5: Antenna of Figure 2. Magnitude of the scattering coefficients of the radiating element fed by the four probes. Reflection coefficients $\left|S_{11}\right|$ at the input port 1 feeding the upper structure (black line), $\left|S_{33}\right|$ at the input port 3 feeding the lower structure (gray line), and transmission coefficient $\left|S_{31}\right|$ from port 1 to port 3 (dashed black line).

In Tables 2 and 3, a summary of the maximum-gain values, locations, and radiation efficiencies is also given for different frequencies, with reference to the feeding of the upper and lower element, respectively. As for the previous unshielded antenna, maximum gain and efficiency is also presented with reference to the alternative choice of the TMM4 for the middle layer (with reduced losses, see Section 2 for details). In particular, while the lower element has a reduced efficiency at higher frequencies, its behavior can be substantially improved with the use of a lower-loss substrate.

4.3. Array Behavior. In order to test further this type of structure, a first simple analysis has been led which gives basic information on the scanning-beam directional features for large arrays. To this aim, the radiation pattern of an array of $140 \times 140$ elements has been computed with an array-factor approximation, for different values of the pointing angle, depending on the selected phase shift between adjacent elements of the array. In Figure 8(a), the pattern on the plane $\varphi=0^{\circ}$ at $5 \mathrm{GHz}$ is shown for a 


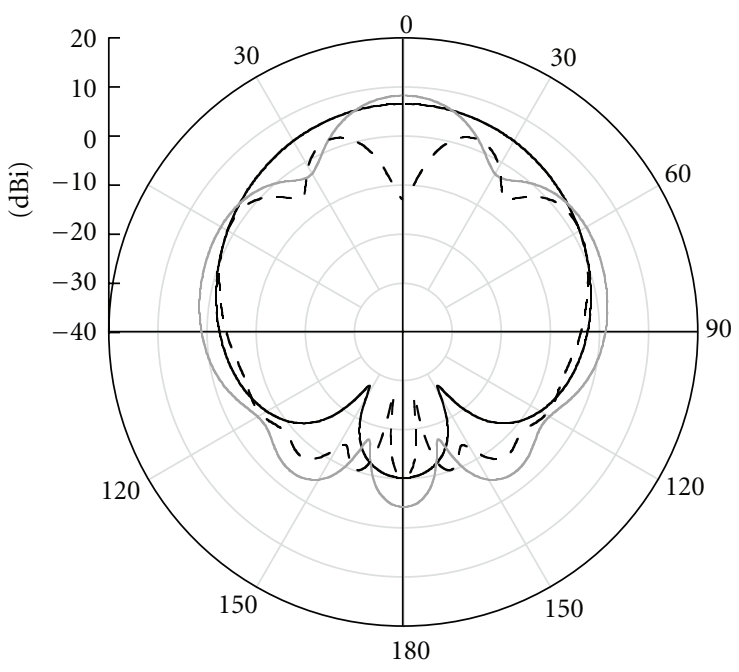

(a)

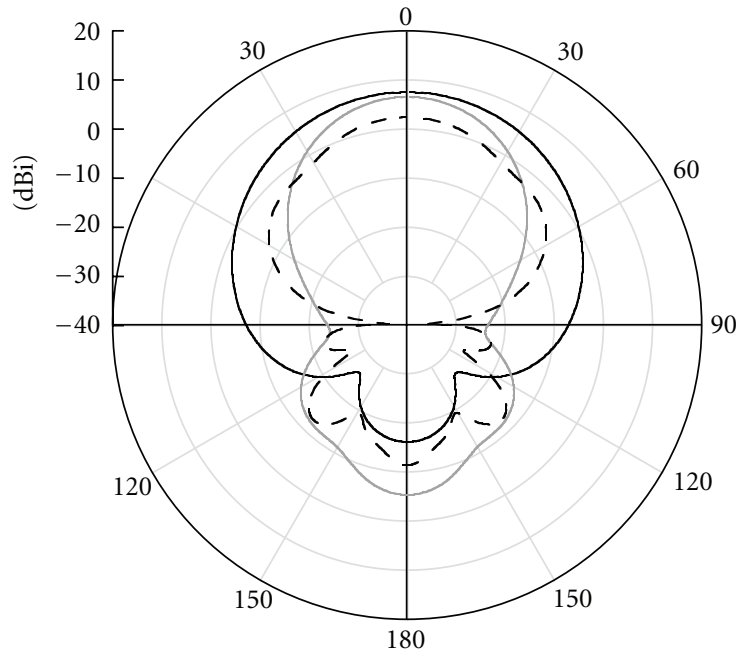

(b)

FIgURE 6: Antenna in Figure 2. Gain in polar form on the principal elevation plane $\phi=0^{\circ}$ of the single element at different frequencies in the operational bandwidth 5-25 GHz: $5 \mathrm{GHz}$ (solid black line), $10 \mathrm{GHz}$ (gray line), $20 \mathrm{GHz}$ (dashed black line). (a) Ports 1 and 2 feed the upper element, exciting a field along the $\theta$ direction. (b) Ports 3 and 4 feed the lower element, exciting a field along the $\varphi$ direction.

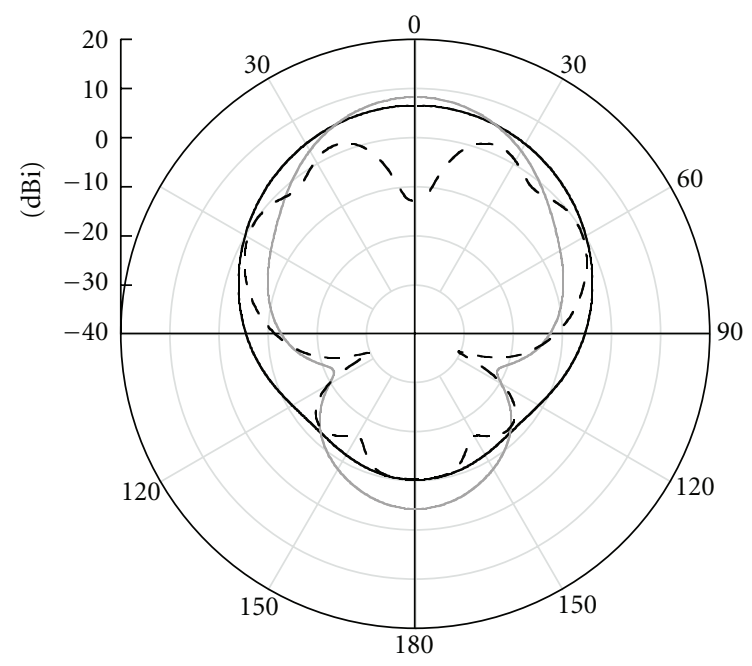

(a)

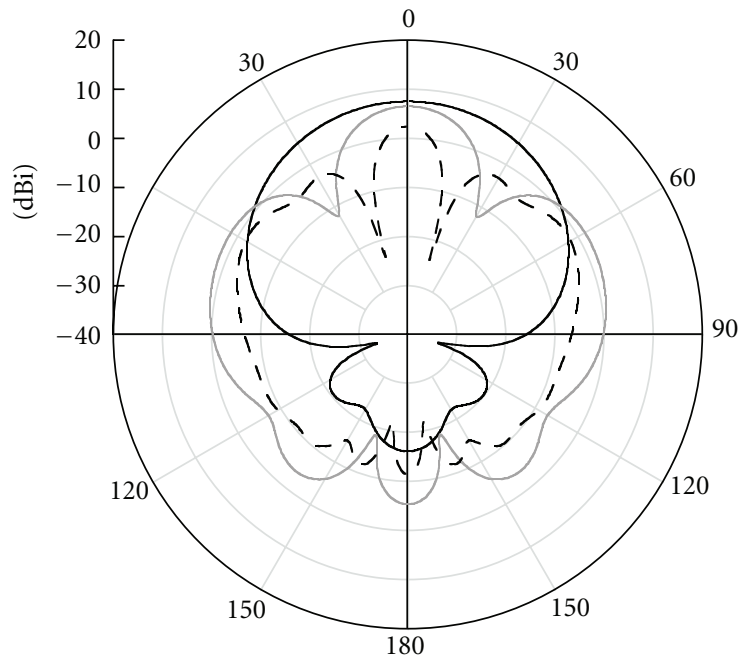

(b)

FIGURE 7: Antenna in Figure 2. Gain in polar form on the principal elevation plane $\phi=90^{\circ}$ of the single element at different frequencies in the operational bandwidth 5-25 GHz: $5 \mathrm{GHz}$ (solid black line), $10 \mathrm{GHz}$ (gray line), $20 \mathrm{GHz}$ (dashed black line). (a) Ports 1 and 2 feed the upper element, exciting a field along the $\theta$ direction. (b) Ports 3 and 4 feed the lower element, exciting a field along the $\varphi$ direction.

broadside radiation when the four probes of each element are fed with common amplitude and a $90^{\circ}$ shift in order to radiate a circular polarized field. The two components along $\theta$ and $\varphi$ are shown in solid black line and in dashed gray line, respectively. In Figure $8(\mathrm{~b})$, the same quantities are computed for a beam pointing at the elevation $\theta=30^{\circ}$ and azimuth $\varphi=0^{\circ}$. The gain at the main lobe direction is fairly regular, around $50 \mathrm{dBi}$; the side-lobe levels are rather reduced (about $20 \mathrm{~dB}$ below the main lobe); the considered phase-scanned pencil beams have half-power beamwidth of about $0.4^{\circ}$. Effects related to grating lobes can be present in connection of the element spacing as phase shift and frequency are varied.

Further results have been obtained considering a linear array made of a small number of cells $(3 \times 1)$ along the $x$ direction in the reference system of Figure 2. In this case, to accurately predict the array performance, a nonapproximate full-wave analysis has been necessary with a proper CAD implementation of the overall physical structure of the three antennas. This rigorous approach allows us to to verify the actual scanning features of the radiated beam as a function of the relevant phase shift. Representative behaviors 
TABLE 2: Maximum gain if ports 1 and 2 are fed (Upper Element).

\begin{tabular}{cccccc}
\hline$f(\mathrm{GHz})$ & Direction $(\vartheta)$ & Max gain & Efficiency & Max gain (low loss) & Efficiency (low loss) \\
\hline 5 & $\vartheta=0^{\circ}$ & $6.5 \mathrm{dBi}$ & $92.9 \%$ & $6.9 \mathrm{dBi}$ & $99.3 \%$ \\
10 & $\vartheta=0^{\circ}$ & $6.5 \mathrm{dBi}$ & $56.1 \%$ & $10.67 \mathrm{dBi}$ & $96.8 \%$ \\
15 & $\vartheta=55^{\circ}, \varphi=70^{\circ}$ & $4.2 \mathrm{dBi}$ & $47.3 \%$ & $7.01 \mathrm{dBi}$ & $88.5 \%$ \\
20 & $\vartheta=50^{\circ}, \varphi=67^{\circ}$ & $2.5 \mathrm{dBi}$ & $40.6 \%$ & $9.04 \mathrm{dBi}$ & $75.0 \%$ \\
25 & $\vartheta=21^{\circ}, \varphi=90^{\circ}$ & $4.2 \mathrm{dBi}$ & $41.5 \%$ & $7.63 \mathrm{dBi}$ & $86.9 \%$ \\
\hline
\end{tabular}

TABLE 3: Maximum gain if ports 3 and 4 are fed (lower element).

\begin{tabular}{cccccc}
\hline$f(\mathrm{GHz})$ & Direction $(\vartheta)$ & Max gain & Efficiency & Max gain (low loss) & Efficiency (low loss) \\
\hline 5 & $\vartheta=0^{\circ}$ & $7.5 \mathrm{dBi}$ & $79.0 \%$ & $8.4 \mathrm{dBi}$ & $95.8 \%$ \\
10 & $\vartheta=0^{\circ}$ & $8.2 \mathrm{dBi}$ & $37.0 \%$ & $10.3 \mathrm{dBi}$ & $88.1 \%$ \\
15 & $\vartheta=61^{\circ}, \vartheta=20^{\circ}$ & $2.5 \mathrm{dBi}$ & $28.0 \%$ & $6.8 \mathrm{dBi}$ & $7.8 \mathrm{dBi}$ \\
20 & $\vartheta=0^{\circ}$ & $2.3 \mathrm{dBi}$ & $28.2 \%$ & $7.5 \mathrm{dBi}$ & $88.6 \%$ \\
25 & $\vartheta=45^{\circ}, \vartheta=20^{\circ}$ & $3.4 \mathrm{dBi}$ & $29.1 \%$ & $80.8 \%$ \\
\hline
\end{tabular}

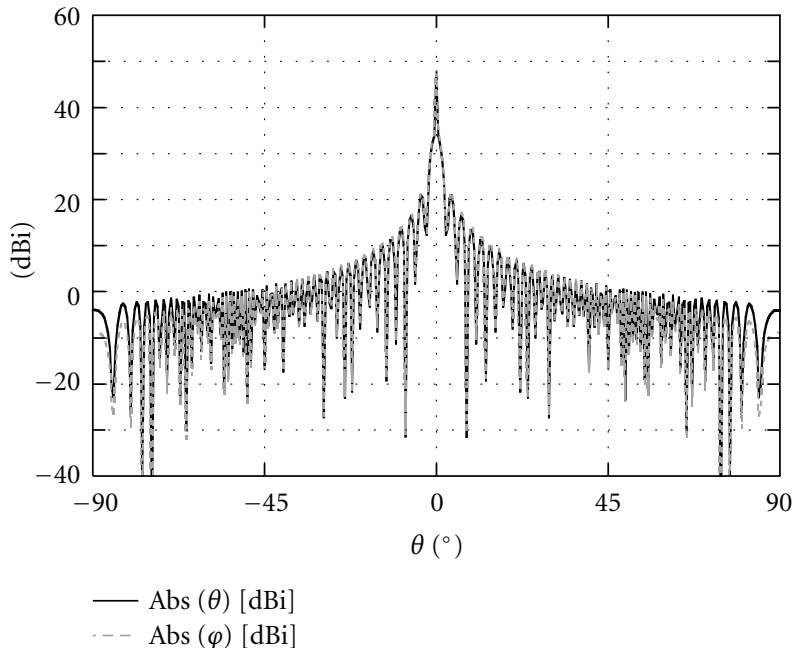

(a)

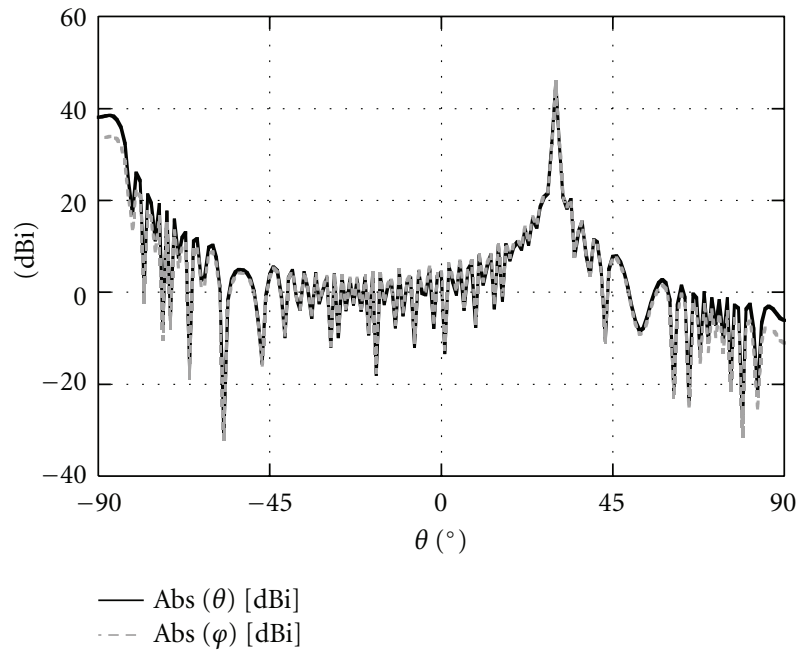

(b)

Figure 8: Array of shielded elements as in Figure 2. Gain on the principal elevation plane $\phi=0^{\circ}$ of a $140 \times 140$ array at 5 GHz. All the ports are fed, exciting a circularly polarized field (its $\theta$ component in solid black line, its $\varphi$ component in dashed gray line). (a) Beam pointing at broadside $\left(\theta=0^{\circ}\right)$. (b) Beam pointing at $\theta=30^{\circ}$.

of radiation patterns for this small array are reported in Figure 9. In Figure 9(a), the gain on the two principal planes is shown when the three upper sets of patches are fed in phase (black curves) and when three lower sets of patches are fed in phase (gray curves). In Figure 9(b), the scanning capabilities of this small array are presented, showing the gain on the principal plane $\varphi=$ when the lower sets of patches are fed, pointing their main beam along the $\varphi=0^{\circ}$ plane, at $\theta=10^{\circ}$, $20^{\circ}, 30^{\circ}, 40^{\circ}$. As expected, a fan beam is obtained, as typical of linear arrays, having maximum gain between 12 and $9 \mathrm{dBi}$.

In Figure 10, couplings among cells are analyzed, again with reference to the feeding of the lower patches, only for the sake of brevity. In Figure 10(a), an input cable feeds the lower patch only in the central cell. The magnitude of the reflection coefficient at this port and of the coupling coefficients with the other cables are shown, avoiding coefficients having equal values due to evident geometrical symmetries. As expected, in the band of frequency investigated, a low level of coupling is found also in this $3 \times 1$ array. In Figure 10(b), the three lower sets of patches of the array are fed in phase through one of the two ports; the magnitude of the active reflection coefficient at the middle cell and at one side cell is reported, proving a good input matching also in this active array configuration.

As a last result, in Figure 11 the radiated patterns of an array made of $3 \times 3$ elements is shown on both the principal planes, $\varphi=0^{\circ}, 90^{\circ}$ for both the upper (Figure 11(a)) and lower (Figure 11(b)) patches. Again, a full-wave analysis has been performed, without recurring to simplified arrayfactor formulations. All the cells of the array are fed in phase 


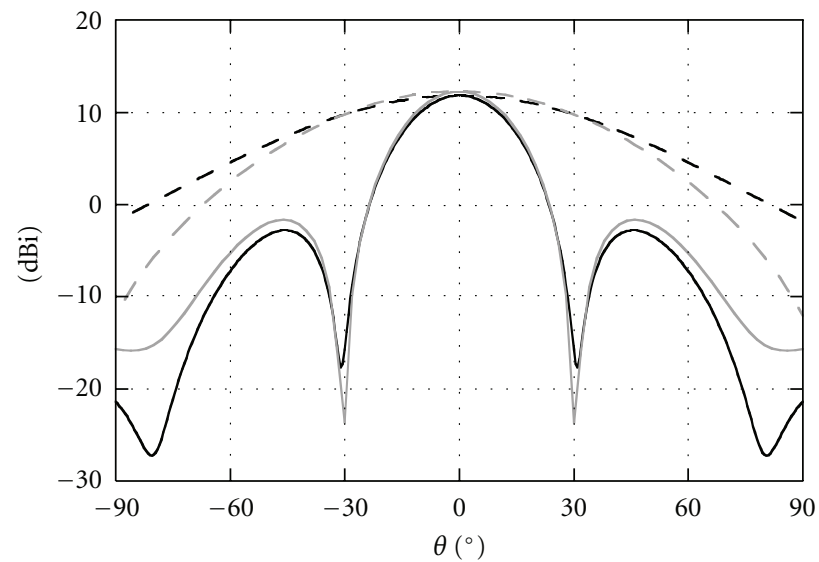

(a)

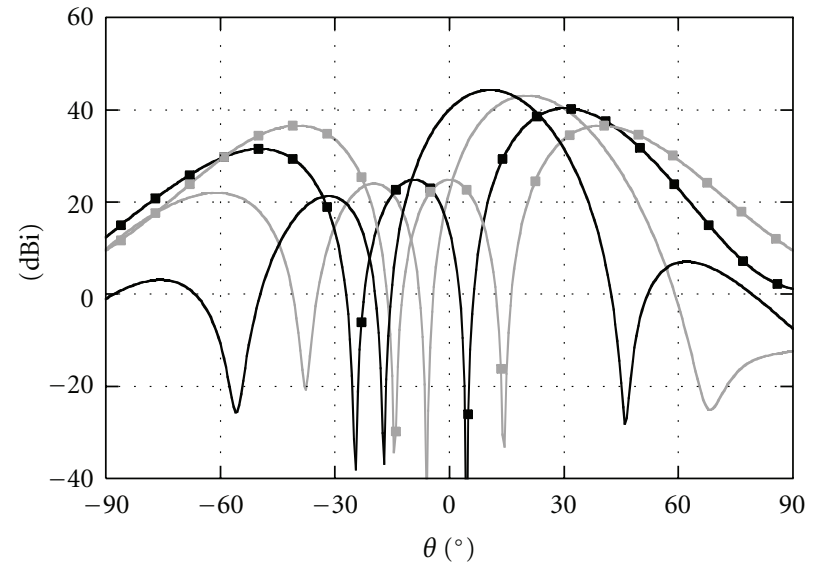

(b)

Figure 9: Array of 3 adjacent shielded cells as in Figure 2 at $5 \mathrm{GHz}$. (a) Gain on the principal elevation planes $\phi=0^{\circ}\left(\right.$ solid lines) and $\phi=90^{\circ}$ (dashed lines). Beam pointing at broadside $\left(\theta=0^{\circ}\right.$, all the cells are excited in phase) by the upper patches (black lines) and by the lower patches (gray lines). (b) The three lower sets of patches are phased in order to point at $\theta=10^{\circ}$ (black line), $\theta=20^{\circ}$ (gray line), $\theta=30^{\circ}$ (black line with squares), and $\theta=40^{\circ}$ (gray line with squares).

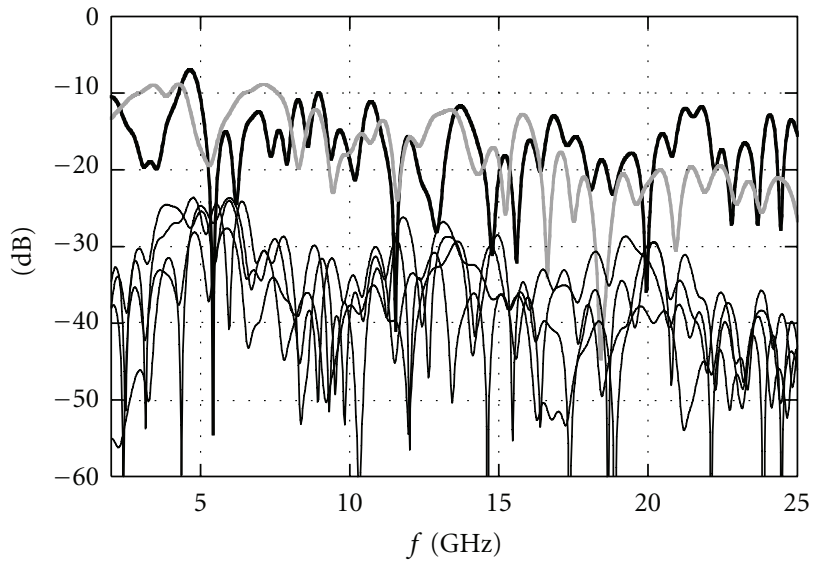

(a)

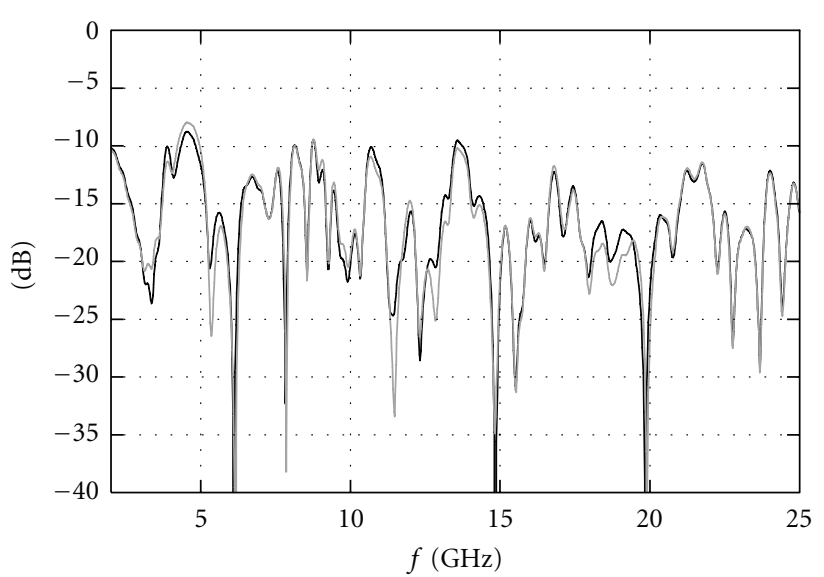

(b)

Figure 10: Array of 3 adjacent shielded cells as in Figure 2 at $5 \mathrm{GHz}$. (a) Only the input port 3 feeds the lower patches in the central cell. Magnitude of reflection coefficient (thick black line), of the coupling coefficient with a cable connected to the upper patch in the same cell (thick gray line), and of coupling coefficients with cables in an adjacent cell (thin black lines). (b) All the three lower sets of patches as fed in phase through port 3 (see Figure 2) and the respective translated ports in the other two cells. Magnitude of the active reflection coefficient versus frequencies at the middle cell of the $3 \times 1$ array (black line) and at one side cell (gray line).

thus radiating a single beam at broadside, with gain around $17 \mathrm{dBi}$, angular width $20^{\circ}$, and sidelobe level $-15 \mathrm{~dB}$ with respect to the maximum gain. As shown in the pictures, a remarkable symmetry of the pattern is obtained on the principal planes, also if the different polarizations are compared.

\section{Conclusion}

A new class of UWB low-cost printed antennas has been presented and optimized for dual-polarized radar applications in the microwave range $5-25 \mathrm{GHz}$. The basic single element is composed by properly fed printed dipoles arranged in rhombic configurations. Different elements can provide almost orthogonal polarizations, depending on their mutual orientation. Attention was paid to practical implementation with inexpensive dielectrics commonly used in PCB technology. These structures are low profile and lightweight and are characterized by high modularity/scalability, which makes them suitable to implement low-profile phased array antennas of various shapes and sizes.

Suitable extensive parametric analyses have been carried out by means of advanced numerical tools as concerns the most efficient choice of the strip geometry configurations. A first design of the antenna element is made with four coplanar rhombic elements, each fed by a probe reaching one end of the central dipole. Relevant results are shown for a single element printed on a laterally unbounded substrate. An advanced alternative design has been proposed, leading 


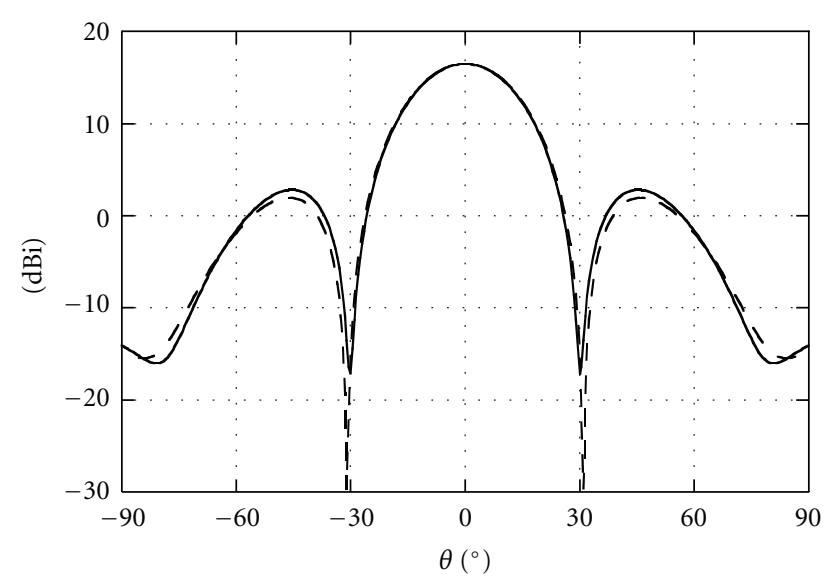

(a)

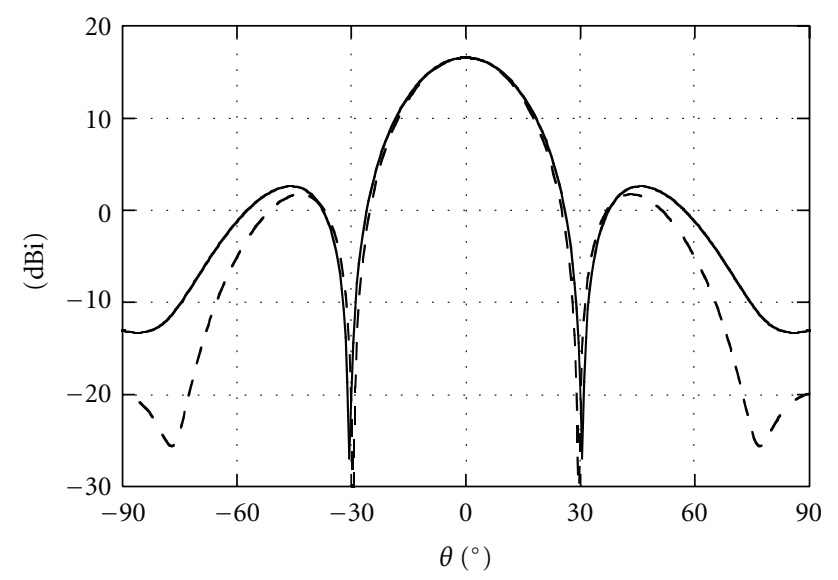

(b)

FIGURE 11: Array of $3 \times 3$ adjacent shielded cells as in Figure 2. Gain on the principal elevation planes $\phi=0^{\circ}$ (solid lines) and $\phi=90^{\circ}$ (dashed lines) at $5 \mathrm{GHz}$. Beam pointing at broadside $\left(\theta=0^{\circ}\right.$, all the cells are excited in phase). (a) The upper patches are fed. (b) The lower patches are fed.

to a shielded radiating element which can be suitably used in array configurations, where the rhombic elements providing the two polarizations are stacked on different substrates. In this structure, lateral metallic walls prevent mutual-coupling effects among elements and reduce possible undesired effects of power leakage in the substrate. A good input matching is obtained in the whole frequency band required, with very promising performance in terms of radiation pattern regularity and gain. The basic performance achievable in array configurations has been finally addressed. On this ground, manufacturing and experimental test of prototypes of $2 \mathrm{D}$ arrays is programmed next.

\section{References}

[1] B. Allen, M. Dohle, E. Okon, W. Malik, A. Brown, and D. Edwards, Eds., Ultra Wideband Antennas and Propagation for Communications, Radar and Imaging, Wiley, 2006.

[2] W. Wiesbeck, G. Adamiuk, and C. Sturm, "Principles of UWB antennas basic properties and design," Proceedings of the IEEE, vol. 97, no. 2, pp. 372-385, 2009.
[3] C.-C. Chen, "Ultrawide bandwidth antenna design," in Antenna Engineering Handbook, J. L. Volakis, Ed., McGrawHill, New York, NY, USA, 2007.

[4] J. J. Lee, "Ultra wideband arrays," in Antenna Engineering Handbook, J. L. Volakis, Ed., McGraw-Hill, New York, NY, USA, 2007.

[5] J. Herd, S. Duffy, M. Weber, G. Brigham, C. Weigand, and D. Cursio, "Advanced architecture for a low cost Multifunction Phased Array Radar," in Proceedings of the IEEE MTT-S International Microwave Symposium Digest (MTT '10), pp. 676-679, Anaheim, Calif, USA, May 2010.

[6] M. Weber, J. Cho, J. Flavin, J. Herd, and M. Vai, "Multifunction phased array radar for U.S. Civil-sector surveillance needs," in Proceedings of the 32nd Conference on Radar Meteorology, pp. 1977-1987, Albuquerque, NM, USA, October 2005.

[7] M. Weber, J. Cho, J. Flavin, and J. Herd, "Multifunction phased array radar: technical synopsis, cost implications and operational capabilities," in Proceedings of the 23rd Conference on International Interactive Information and Processing Systems (IIPS) for Meteorology, Oceanography and Hydrology, San Antonio, Tex, USA, January 2007.

[8] R. Waterhouse, Ed., Printed Antennas for Wireless Communications, Wiley, New York, NY, USA, 2007.

[9] V. N. Bringi and V. Chandrasekar, Polarimetric Doppler Weather Radar: Principles and Applications, Cambridge University Press, Cambridge, UK, 2001.

[10] D. R. Jackson, "Microstrip antenna," in Antenna Engineering Handbook, J. L. Volakis, Ed., McGraw-Hill, New York, NY, USA, 2007.

[11] C. D. Nallo, F. Mesa, and D. R. Jackson, "Excitation of leaky modes on multilayer stripline structures," IEEE Transactions on Microwave Theory and Techniques, vol. 46, no. 8, pp. 10621071, 1998.

[12] P. Baccarelli, P. Burghignoli, F. Frezza, A. Galli, G. Lovat, and D. R. Jackson, "Approximate analytical evaluation of the continuous spectrum in a substrate-superstrate dielectric waveguide," IEEE Transactions on Microwave Theory and Techniques, vol. 50, no. 12, pp. 2690-2701, 2002.

[13] K. Ghorbani and R. B. Waterhouse, "Dual polarized wideband aperture stacked patch antennas," IEEE Transactions on Antennas and Propagation, vol. 52, no. 8, pp. 2171-2174, 2004.

[14] D. Tallini, A. Galli, M. Ciattaglia, L. Infante, A. De Luca, and M. Cicolani, "A new low-profile wide-scan phased array for UWB applications," in Proceedings of the European Conference on Antennas and Propagation, pp. 1-5, Edinburgh, UK, November 2007.

[15] A. Galli, G. Valerio, D. Tallini, A. De Luca, and M. Cicolani, "Optimization of multifunctional UWB planar phased arrays," in Proceedings of the European Conference on Antennas and Propagation, pp. 571-574, Berlin, Germany, March 2009.

[16] S. Mazzocchi, G. Valerio, M. Zucca, M. Ciattaglia, A. De Luca, and A. Galli, "New multifunctional UWB planar antenna based on printed dipoles in rhombic configuration," in Proceedings of the European Conference on Antennas and Propagation, Barcelona, Spain, April 2010.

[17] A. A. Qureshi, M. U. Afzal, T. Taqueer, and M. A. Tarar, "Performance analysis of FR-4 substrate for high frequency microstrip antennas," in Proceedings of the China-Japan Joint Microwave Conference (CJMW'11), pp. 159-162, April 2011.

[18] http://www.modefrontier.com. 
[19] C. Poloni and V. Pediroda, "GA coupled with computationally expensive simulations: tools to improve efficiency," in Algorithms and Evolution Strategies in Engineering and Computer Science, pp. 267-288, Wiley, 1997.

[20] CST Microwave Studio Manual, CST, Germany, 2002. 

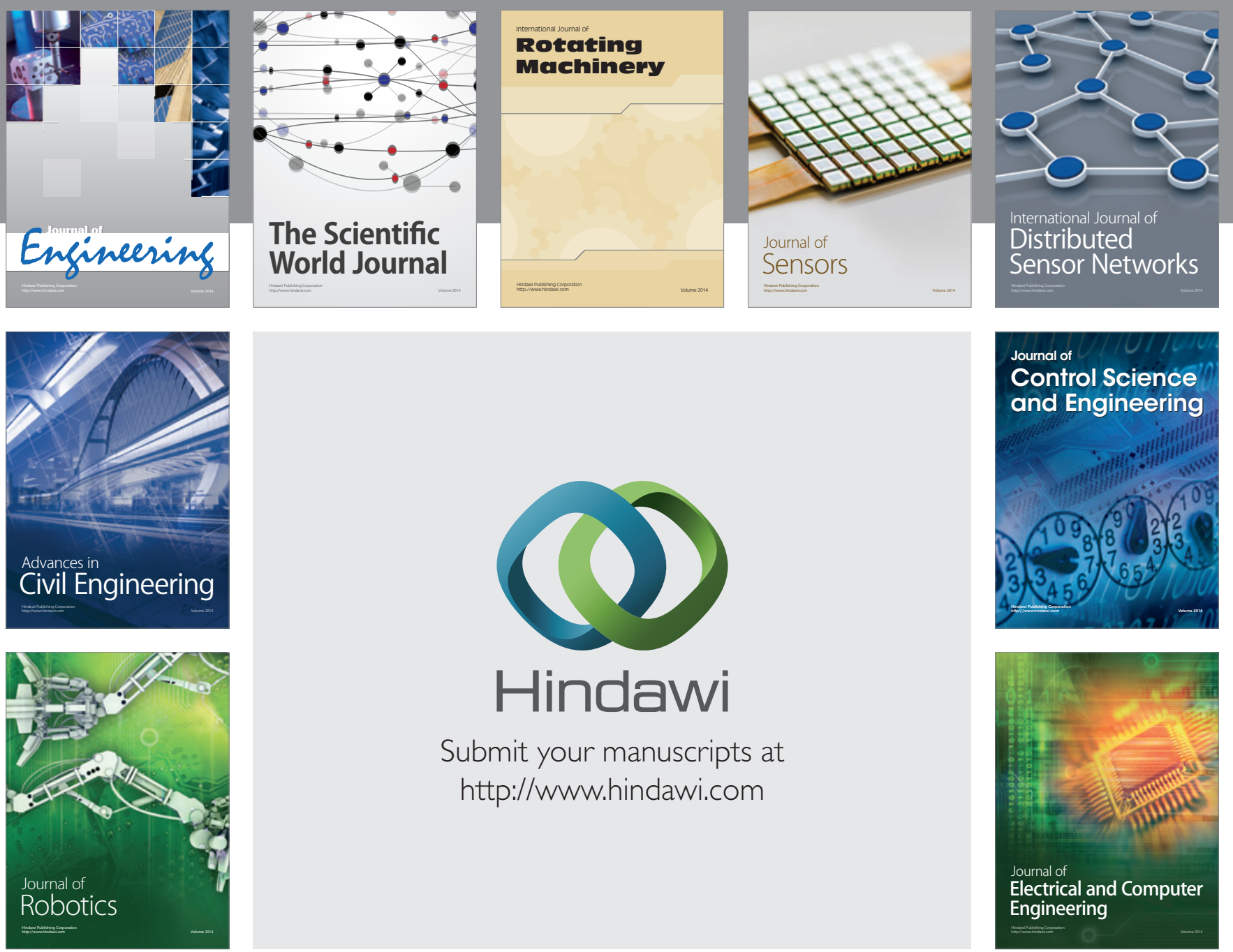

Submit your manuscripts at

http://www.hindawi.com
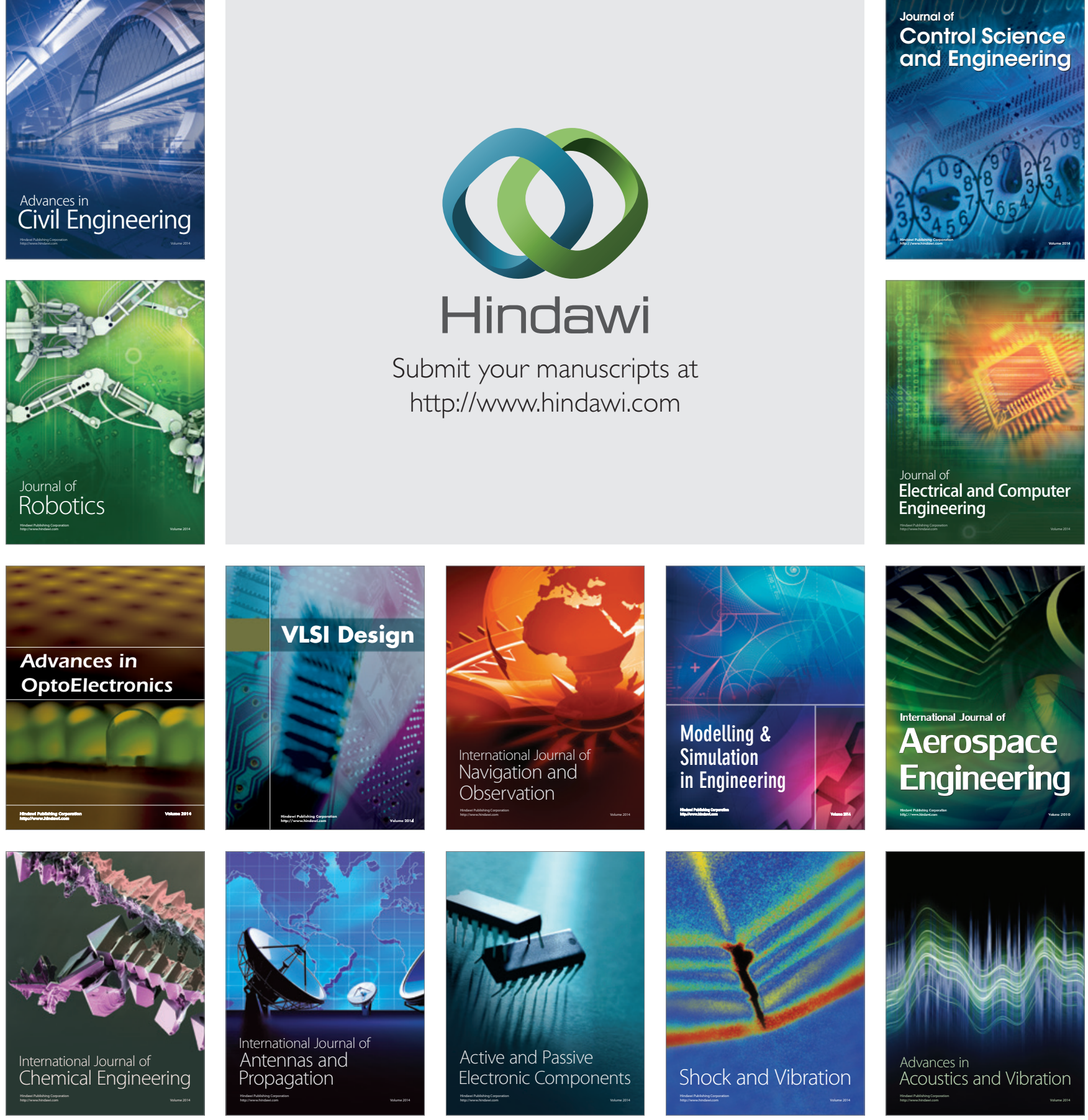